\title{
Comparación de tres sitios anatómicos de extracción de muestras de medula ósea para el diagnóstico de leishmaniosis canina
}

\author{
Maidana, H.R..; Llano, E.G.. ${ }^{2}$; Báez, A.D. ${ }^{3}$; Cabrera, W.R. ${ }^{2}$; López, J.E. ${ }^{3}$ \\ Cátedras Clínica de Pequeños Animales ${ }^{(1)}$, Anatomía II (2) y Hospital de Clínicas ${ }^{(3)}$, Facultad de Ciencias \\ Veterinarias, UNNE, Sargento Cabral 2139, Corrientes (3400), Argentina. Tel. 03783425753. \\ E-mail: anato2@vet.unne.edu.ar.
}

\begin{abstract}
Resumen
Maidana, H.R.; Llano, E.G.; Báez, A.D.; Cabrera, W.R.; López, J.E.: Comparación de tres sitios anatómicos de extracción de muestras de médula ósea para el diagnóstico de leishmaniosis canina. Rev. vet. 21: 2, 144-147, 2010. El objetivo del trabajo fue comparar tres lugares de extracción de médula ósea: esternón, cresta ilíaca y tercio ventral de las últimas costillas, estableciendo las ventajas y dificultades anatómicas, el volumen de muestra obtenida, la tolerancia del paciente y la celularidad de cada región. Se realizaron extracciones de muestras de médula ósea a 10 perros, con las cuales se efectuaron frotis que se fijaron con alcohol y se colorearon con Giemsa. Mediante microscopía óptica, a través del objetivo de inmersión se identificó la cantidad y los tipos celulares hallados en cada una de las regiones muestreadas. El lugar de elección resultó ser el tercio ventral de las costillas X, XI y XII, debido a que la situación superficial de las costillas permite la correcta fijación de las mismas y el guiado de la aguja, minimizando los riesgos de lesiones accidentales a otros órganos. Además, la condición delgada del hueso cortical permite allí una mejor penetración de la aguja y asegura la obtención de un volumen suficiente de muestra para realizar el extendido. La punción es bien tolerada y con la práctica se convierte en una técnica rápida y segura para el animal y el operador.
\end{abstract}

Palabras clave: perro, leishmaniosis, diagnóstico, médula ósea.

\begin{abstract}
Maidana, H.R.; Llano, E.G.; Báez, A.D.; Cabrera, W.R.; López, J.E.: Comparison of three anatomical places of bone marrow sampling for the diagnosis of canine leishmaniosis. Rev. vet. 21: 2, 144-147, 2010. The objectives of this research were to compare three extraction sites of canine bone marrow: sternum, iliac crest and ventral third of the ribs, and to evaluate advantages and anatomical difficulties, volume of the sample, patient's tolerance and cellularity of each region. For this purpose, 10 dogs underwent bone marrow harvesting. Smears were fixed with alcohol, stained with Giemsa, and observed by means of light microscopy with immersion objective to identify cell type for each sampled region. The ventral third of the ribs X, XI and XII may be considered the best site to obtain bone marrow samples, because the superficial position of ribs makes them easy to be located and allows the correct guiding of the needle, minimizing the risks of accidental injury to other organs. Also, the thin cortical bone layer allows better penetration of the needle and a sufficient volume of sample to prepare the smear. The needle puncture is well tolerated and the practice becomes a quick and safe technique for both animal and operator.
\end{abstract}

Key words: dog, leishmaniosis, diagnosis, bone marrow.

\section{INTRODUCCIÓN}

La leishmaniosis es una enfermedad zoonótica que afecta a las personas y animales, producida por un parásito del genero Leishmania. Los caninos pueden padecer las formas cutáneas, mucocutáneas y visceral, con signos clínicos muy variables. En la actualidad se

Recibido: 28 setiembre 2010 / Aceptado: 18 octubre 2010 considera que los perros en las comunidades son el reservorio principal de la leishmaniosis visceral en seres humanos ${ }^{2}$.

Las leishmanias pertenecen a la familia Trypanosomatidae, habiéndose identificado gran número de especies y subespecies. Leishmania chagasi es el protozoario que produce leishmaniosis visceral, siendo transmitido en América por la picadura del flebótomo Lutzomyia sp., pequeño insecto de 2 a $3 \mathrm{~mm}$ de largo ${ }^{10,12}$. 
Otras especies de Leishmania que pueden parasitar a perros y seres humanos son L. braziliensis y L. trópica entre otras.

Ocasionalmente, se han descrito casos autóctonos en países en los que no hay flebótomos. Se conoce que un perro nacido en una zona endémica de una madre afectada desarrolló la enfermedad, sugiriendo la posibilidad de transmisión intrauterina. También se ha señalado que la enfermedad se puede transmitir como resultado de una transfusión de sangre proveniente de animales infectados ${ }^{4}$.

La enfermedad, en sus distintas presentaciones, no tiene predilección por edad, raza o sexo de los animales involucrados, ni discrimina la condición socioeconómica de los propietarios, aunque en nuestro país la mayoría de las veces se la halla relacionada con los sectores marginales y carenciados de la población ${ }^{1}$.

El mecanismo de infección requiere que el vector ingiera sangre al picar a un vertebrado infectado con los parásitos en estadio de amastigotes, los que se multiplican en el intestino del insecto transformándose en promastigotes. Al picar a los vertebrados, el flebótomo los inocula por regurgitación. Las leishmanias son internalizadas por los macrófagos y otras células dendríticas. Pueden vivir en las células retículoendoteliales del huésped porque neutralizan el $\mathrm{pH}$ y detoxifican los metabolitos oxigenados. En los macrófagos, los parásitos se multiplican por fisión binaria hasta que rompen la célula y se diseminan a otros macrófagos ${ }^{12}$.

Los animales pueden desarrollar una enfermedad progresiva con un desenlace fatal, o permanecer asintomáticos. El período de incubación es muy largo, de un mes a siete años. En este período los microorganismos se diseminan ampliamente, con predilección por la médula ósea, nódulos linfáticos, bazo e hígado.

Los signos clínicos que se presentan incluyen debilidad, lesiones cutáneas, pérdida de peso y letargia. Las causas de la letargia deberían investigarse en la anemia, atrofia muscular, poliartropatía e insuficiencia renal crónica, todo lo cual conduce a la disminución de las masas musculares. Se han observado caninos con claudicaciones atribuidas al crecimiento exagerado de las uñas y a la poliartritis autoinmune, polimiositis y lesiones óseas, en las que se encuentran los parásitos en grupos de lesiones inflamatorias granulomatosas $6,9,14$.

Los parásitos se multiplican en los macrófagos del hígado, produciendo una hepatitis crónica activa y ocasionalmente un aumento palpable del hígado, vómitos, poliuria, polidipsia, anorexia y pérdida de peso. La enteritis puede ser el resultado del daño parasitario directo (enteritis granulomatosas) o consecuencia de la insuficiencia renal. También se ha descrito un caso de pancreatitis hemorrágica aguda ${ }^{8}$. Frecuentemente se reportan epistaxis, generalmente unilaterales, resultado de las lesiones ulcerosas de la mucosa nasal y/o de la alteración de la coagulación debida a hiperglobulinemia y trombocitopenia. La linfoadenopatía, local o generalizada, es uno de los signos más consistentes, aunque ocasionalmente puede estar ausente. Las lesiones oculares afectan principalmente el segmento anterior del ojo, la dermatitis periorbital y la blefaritis asociada son los signos clínicos más comunes ${ }^{13}$. La linfoadenopatía generalizada y la hepatoesplenomegalia son hallazgos comunes ${ }^{3}$.

Para arribar al diagnóstico es necesario realizar una minuciosa exploración del paciente, obteniendo un diagnostico presuntivo que se confirma a través de métodos complementarios. Ninguna prueba tiene una efectividad del 100\% y se obtuvieron resultados falsos negativos en más del 10\% de los perros parasitados. El diagnóstico clínico de leishmaniosis visceral canina es difícil, debido a que los síntomas son muy variables y pueden ser semejantes a los de otras enfermedades ${ }^{6,7}$. Entre las pruebas diagnósticas pueden citarse la técnica serológica con la tira Kalazar Detect (Canine) y el examen parasitológico del material obtenido por punción de médula ósea y nódulos linfáticos, o raspado de lesiones de piel $^{5,7,11}$.

El objetivo del trabajo fue comparar tres lugares de extracción de medula ósea: esternón, cresta ilíaca y tercio ventral de las últimas costillas, teniendo en cuenta las ventajas y dificultades anatómicas, el volumen de muestra obtenida, la evaluación subjetiva de la tolerancia del paciente mediante la observación de movimientos voluntarios frente a la aplicación de un estímulo (punción con aguja 25/8 atravesando los diferentes planos anatómicos) y la celularidad obtenida en cada región en particular.

\section{MATERIAL Y MÉTODOS}

Se utilizaron 10 perros de ambos sexos, de diferentes razas, tamaños y edades. Para abordar cada uno de los sitios a comparar, el animal fue sujetado en decúbito lateral, tras lo cual se identificó la región anatómica de extracción y se realizó la correspondiente antisepsia. Luego se introdujo una aguja 25/8 atravesando los planos superficiales y la capa cortical del hueso. Al llegar a la cavidad medular, se acopló una jeringa de $5 \mathrm{ml}$ realizando una aspiración sostenida para obtener la mayor cantidad de muestra posible. Seguidamente se realizó un frotis con el material extraído, fijándose con alcohol metílico y coloreándose durante 15 min con Giemsa (1 $\mathrm{ml}$ en $10 \mathrm{ml}$ de agua). La observación por microscopía óptica se realizó con objetivo de inmersión, evaluándose la cantidad y tipos celulares hallados en cada región muestreada.

\section{RESULTADOS Y DISCUSIÓN}

En las punciones esternales (Figura 1) el abordaje anatómico fue dificultoso, la reacción del animal fue considerable (la evaluación subjetiva de la tolerancia indicó una mayor sensibilidad), el volumen de la muestra obtenida y la celularidad fueron escasos (Figura 2), apareciendo gran cantidad de eritrocitos y algunos leucocitos (60\% polimorfonucleares y $40 \%$ monomorfonucleares). 
Tabla 1. Comparación entre los tres lugares de extracción evaluados.

\begin{tabular}{lccc}
\hline característica & esternón & cresta ilíaca & costilla \\
\hline abordaje anatómico & dificultoso & bueno & muy bueno \\
reacción del paciente & considerable & considerable & leve \\
cantidad de muestra & escasa & abundante & intermedia \\
celularidad & escasa & escasa & escasa \\
\hline
\end{tabular}

A nivel de la cresta iliaca (Figuras 3 y 4) las dificultades anatómicas para el abordaje medular fueron menores que en el sitio anterior, el volumen de muestra obtenida fue considerablemente mayor y la tolerancia del paciente, así como la celularidad observada al mi-

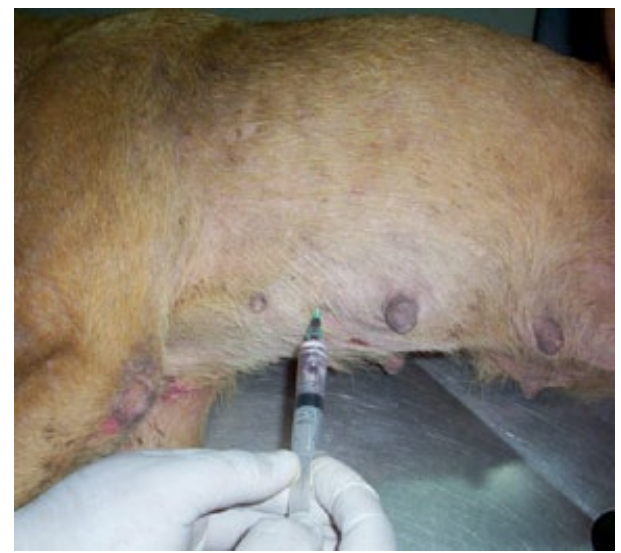

Figura 1. Extracción por vía esternal.

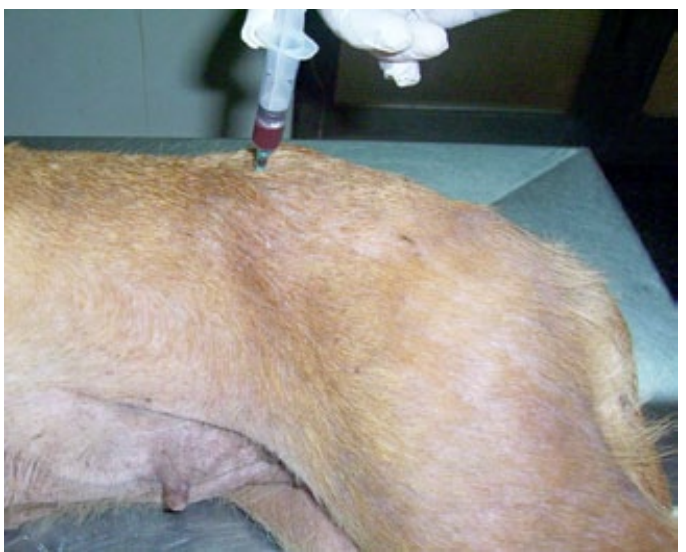

Figura 3. Abordaje de la cresta ilíaca.

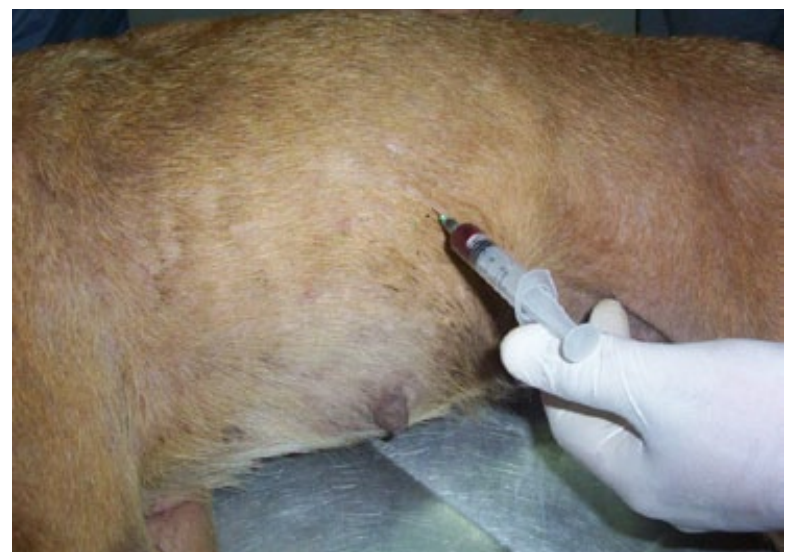

Figura 5. Extracción por vía costal. croscopio fueron similares al del abordaje esternal (Figuras 3 y 4 ).

En el tercio ventral de las últimas costillas (Figuras 5 y 6 ) las dificultades anatómicas fueron menores, el volumen de muestra fue intermedio, la tolerancia del paciente fue mayor (reacción muy leve) y la celularidad observada fue similar a la de las regiones mencionadas anteriormente.

La Tabla 1 muestra un resumen de los resultados obtenidos en cada sitio anatómico de extracción de médula ósea. Surge como conclusión que el tercio ventral de las costillas X, XI y XII califican como el lugar de

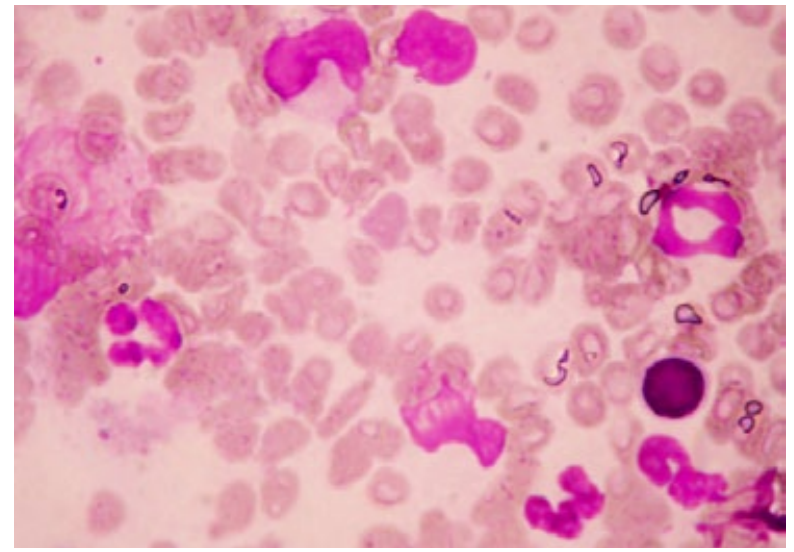

Figura 2. Frotis del material extraído de esternón.

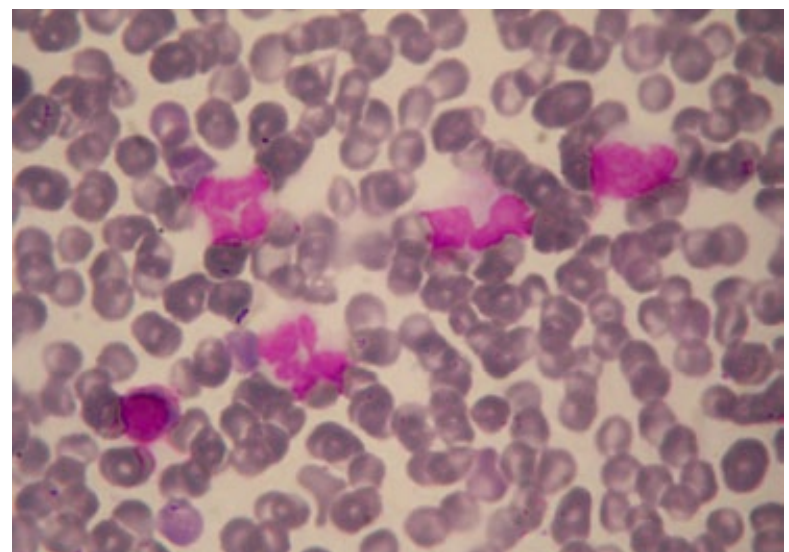

Figura 4. Células provenientes de cresta ilíaca.

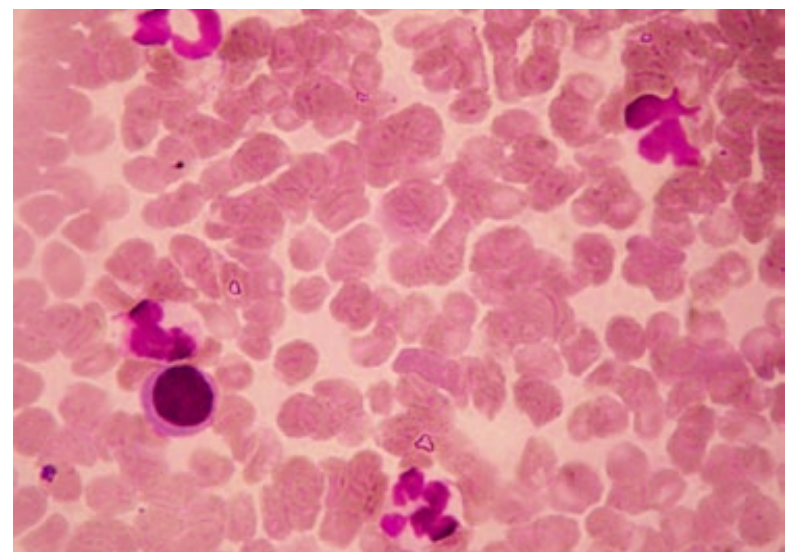

Figura 6. Celularidad de la muestra costal. 
elección para el abordaje, debido a varios motivos. En primer lugar, la ubicación superficial de las costillas permite una correcta fijación de las mismas y posibilita guiar la aguja minimizando los riesgos de lesiones accidentales a otros órganos. La condición delgada de la capa cortical permite una más fácil penetración de la aguja y asegura la obtención de un volumen suficiente de muestra. En este sitio la punción fue bien tolerada por el animal. Con la práctica, esta técnica se convierte en una maniobra rápida y segura para el animal y el operador.

Agradecimientos. Al Dr. Eduardo Pérez Valega por su valioso asesoramiento. A la Lic. Mirna Mabel Zernetti por traducir al inglés el resumen del trabajo.

\section{REFERENCIAS}

1. Borda CE, Rea MF, Rosa JR, Mosqueda LA. 1999. Leishmaniasis en la ciudad de Corrientes, Argentina. Memorias del XIV Congreso Latinoamericano de Parasitología, Acapulco (México), p. 14.

2. Bravo L, Frank LA, Brenneman KA. 1993. Canine leishmaniasis in the United States. Comp Contin Educ 15: 699-705.

3. Ciarantella P, Oliva T, De Luna R. 1997. A retrospective clinical study of canine leishmaniasis in 150 dogs naturally infected by Leishmania infantum. Vet Rec 141: 539-543.

4. Diaz Espineira M, Slappendel R. 1997. A case of autochtthonous canine leishmaniasis in the Netherlands. Vet $Q$ 19: 69-71.

5. Fernández G, Maidana H, Pérez Valega E, Maccio O, Gorodner J. 2004. Examen parasitológico directo en el estudio de la leishmaniasis tegumentaria en animales y humanos, descripción y análisis. Memorias de las Comunicaciones Cientificas y Tecnológicas UNNE, Corrientes (Argentina), V-011.
6. Ferrer L, Rabanal F, Fondevila D, Ramos J, Domingo M. 1988. Skin lesions in canine leishmaniasis. J Small Anim Pract 29: 381-388.

7. Ferrer L, Aisa MJ, Roura X, Porras M. 1995. Serological diagnosis and treatment of canine leishmaniasis. Vet Rec 136: 514-516.

8. Font A, Closa JM, Molina A, Mascort J. 1993. Thrombosis and nephrotic syndrome in a dog with visceral leishmaniasis. J Small Anim Pract 34: 466-470.

9. Font A, Roura X, Fondevila D. 1996. Canine mucosal leishmaniasis. J Am Anim Hosp Ass 32: 131-137.

10. Hommel M. 1978. The genus Leishmania: biology of the parasites and clinical aspects. Bull Inst Pasteur 75: 5-12

11. Maidana HR, Llano EG, Cabrera WR, Báez AD, Burna AN, Pérez Valega E, Gorodner J. 2009. Lugar de elección en la toma de muestras de medula ósea para el diagnostico de leishmaniasis en caninos. Memorias de las Comunicaciones Científicas y Tecnológicas UNNE, Resistencia (Argentina), CV 006.

12. Ministerio de Salud, Presidencia de la Nación Argentina. 2010. Leishmaniasis visceral. Guía para el equipo de Salud, Ed. Dirección de Epidemiología, Buenos Aires, 52 p.

13. Roze M. 1986. Manifestations oculaires de la leishmaniose canine. Recueil Méd Vétér 162: 19.

14. Spreng D. 1993. Leishmanial polyarthritis in two dogs. $J$ Small Anim Pract 34: 559-563. 